\title{
Magnetic Resonance Imaging of Metal on Metal Implants: A Breakthrough
}

\author{
Pilania K, Jankharia B \\ Department of Radio diagnosis and Imaging, Jankharia Imaging Centre, Mumbai, India
}

\begin{abstract}
Background: Metal-on-metal (MoM) hip replacement, a durable alternative to conventional metal -on-polyethylene hip replacement, is increasingly common nowadays. There is a 4-8\% incidence of pain and complications which usually go undetected on radiographs. MRI has a vast potential for early diagnosis in these cases. Aim: To evaluate the feasibility of MR imaging in MoM hips and to review the MR imaging features of various complications associated with metal on metal hip implants. Materials and Methods: 41 patients with 52 replaced hips using MoM prostheses underwent MRI on a $1.5 \mathrm{~T}$ scanner using standard clinical protocols optimized to minimize metallic susceptibility artifact, known as metal artefact reduction sequences (MARS). A standardized reporting was done with specific comment on periprosthetic osteolysis, loosening, periprosthetic soft tissue mass, gluteal muscle atrophy, iliopsoas atrophy, muscle edema, muscle/ tendon tear and lymphadenopathy. Results: Diagnostic images were obtained in all patients. Of the 52 hips, 32 had total hip replacement (THR) and 20 had resurfacing. The following abnormalities were found. Periprosthetic osteolysis (5), Periprosthetic soft tissue mass (24), Gluteal muscle atrophy moderate to severe (15), Iliopsoas atrophy (15), Muscle edema (0), Muscle / tendon tear (0), Lymphadenopathy (5). Conclusion: MRI on a $1.5 \mathrm{~T}$ scanner is a viable technique to image the MoM hip and shows well the various complications associated with post arthroplasty hips.
\end{abstract}

Keywords: Hip replacement, Metal-on-metal implant, Post arthroplasty complications.

\section{Introduction}

Metal-on-metal (MoM) hip replacement, a durable alternative to conventional metal-onpolyethylene hip replacement, is increasingly common nowadays and with increasing

Corrospondence to: Dr. Khushboo Pilania, MD, Radiodiagnosis, Department of Radiodiagnosis and Imaging, Jankharia Imaging Centre, Mumbai, India. E-mail:- pilania.khushboo@gmail.com life span and decreased clinical threshold for surgery this number will continue to rise. It can either have a conventional total hip replacement or the newer resurfacing design which has the advantage of sparing thenative femoral neck. The common indications are avascular necrosis and osteoarthritis, with seronegative arthritides, rheumatoid arthritis, trauma and infection being among the other common indication. 
There is a $4-8 \%$ incidence of pain and complications which are usually asymptomatic but may require surgical revision and hence highlights the need for imaging followup. The various complications following arthroplasty include: Aseptic loosening, particle disease (osteolysis), infection, component wear, dislocation, fracture, heterotopic ossification, metalinduced reactive mass, abductor muscle tear, iliopsoas impingement and muscle atrophy.

Radiographs, though still the mainstay in evaluating a post arthroplasty hip, and reasonably reliable in the detecting dislocation, fracture and heterotopic ossification, has vast limitations especially in evaluation of soft tissue details. Differentiating loosening from infection especially in their early stages can also be tricky on radiographs and MR complements radiographs perfectly. Nevertheless, cross sectional imaging in postarthroplasty hips has always been a challenge because of the susceptibility artifacts on MRI and beam hardening on CT scan which prevent accurate evaluation of regions of interest near the implants. ${ }^{1,2}$ However, lately with the advent of specialised metal artefact reduction sequences(MARS) and newer instrumentation, the challenge of susceptibility artefacts due to the metal implants have largely been overcome and MR with its inherent ability of better soft tissue delineation has provided a phenomenal edge in providing a more accurate diagnosis of the underlying complications. ${ }^{3,4}$

Magnetic susceptibility effects on spins near a metal object in a magnetic field result in two main types of image distortion: (a) due to spatial misregistration, on both conventional spin-echo (SE) and gradient- echo (GRE) images, and (b) loss of signal intensity because of intravoxel dephasing, (T2*effect) on GRE images ${ }^{1,3}$ as these sequences, unlike SE, include no $180^{\circ}$ refocusing pulse, which enables recovery of the transverse signal lost because of static magnetic field inhomogeneities and bulk susceptibility differences in SE sequences. $4-7$

The metal artifact reduction sequences (MARS) reduce the size and intensity of susceptibility artifacts from magnetic field distortion thus allowing assessment of the periprosthetic soft tissues. These sequences, which are based on view angle tilting, smaller interecho spacing, increased readout bandwidth and frequency-encoding direction oriented away from the tissues of interest, can conveniently be used in conjunction with any spin-echo sequence and requires minimal additional imaging time..$^{6-15}$

Despite this, MR imaging in MoM hip replacements has been relatively unexplored and the purpose of this study is to show that MRI is feasible in patients with MoM hips and to provide an insight into the MR imaging features of the various complications associated with MoM hips.

\section{Materials and Methods}

41 patients with 52 replaced hips using MoM prostheses presented for MRI. The duration of replacement ranged from 6 months to 5.5 years.

All MRI studies were performed on a $1.5 \mathrm{~T}$ scanner (Magnetom, Siemens, Germany) after obtaining an informed consent from the patients. Both hips were scanned together in a six channel body coil. All subjects 
underwent MRI using standard clinical protocols optimized to minimize metallic susceptibility artefact. The protocol included STIR, T1W and T2W coronal, T1W and T2W axial and T2W sagittal sequences. Ethical committee clearance was not obtained as the study was retrospective.
Fast spin-echo (FSE) T1W and T2W images were obtained in the coronal, axial and sagittal planes with the frequency encoding direction oriented away from the tissues of interest. The parameters were as follows: TR range, 400-4000 ms; TE range, 8-102 ms; bandwidth 600 - 751; FOV, 300-400mm; number of signals acquired, 2; acquisition

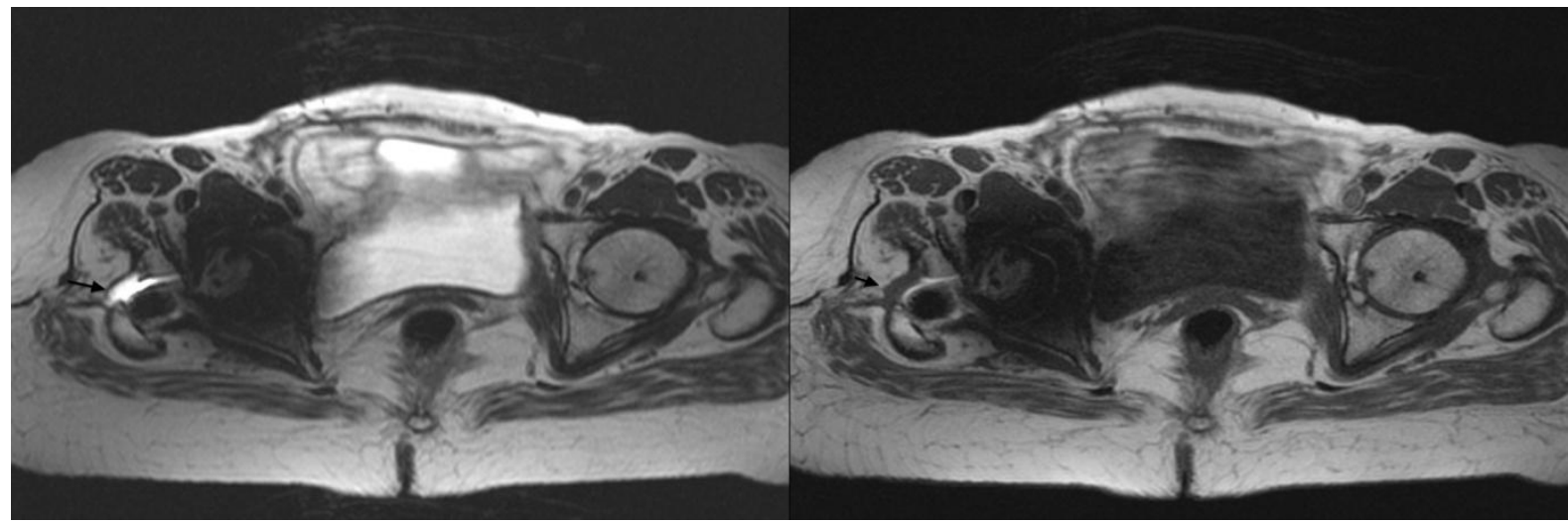

Fig. 1: Type 1 collection anterior to greater trochanter.

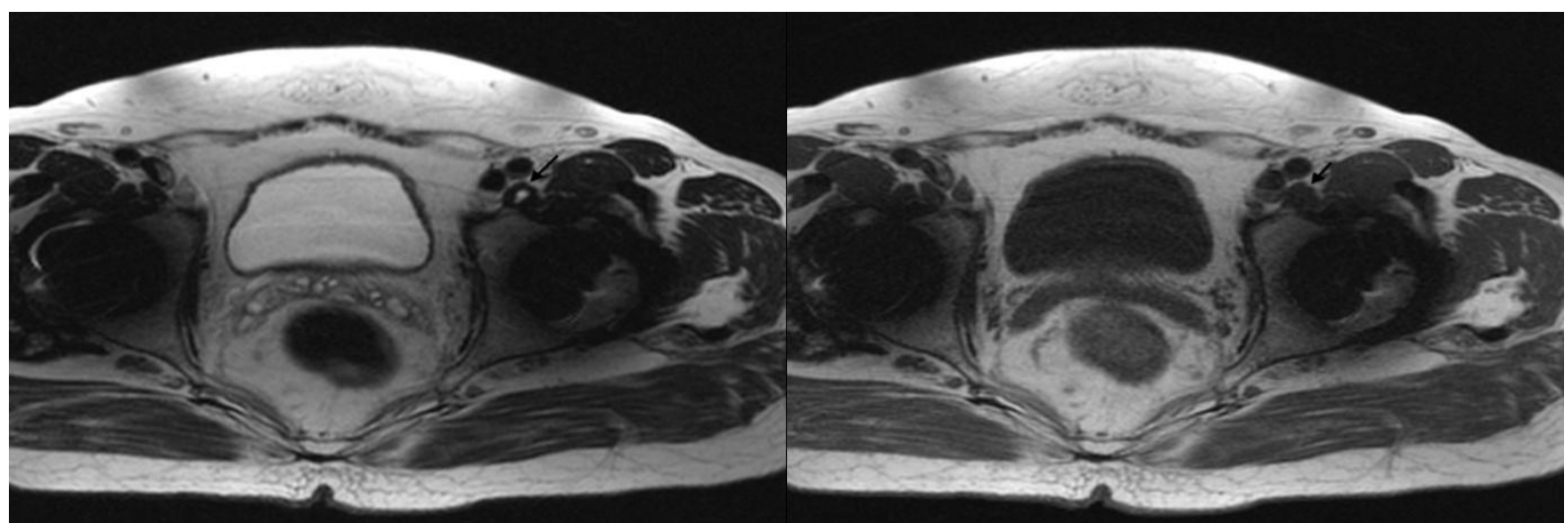

Fig. 2: Thick walled type 2 collections in left psoas distally.



Fig. 3: Type 3 collection: Heterogenous signal intensity soft tissue within the iliopsoas bursa on left. 
matrix, 256-320 (frequency) $\times$ 75-80(phase); and slice thickness, $4 \mathrm{~mm}$. A fast inversion recovery sequence was performed in the coronal plane using the following parameters: TR/ TE, 4480/49; inversion time, 130 ms; bandwidth, 407; FOV, 300$350 \mathrm{~mm}$; number of signals acquired, 2; acquisition matrix, 256 (frequency) $\times 100$ (phase); and slice thickness, $4 \mathrm{~mm}$. The average acquisition time was 2-4 min for each imaging plane with a total acquisition time of approximately 25-30 minutes. The images from each sequence were loaded on a workstation (Osirix, IMAC, Apple, Inc).

Structured reporting was done with comments on specific issues following hip replacement viz, Periprosthetic soft tissue mass, Periprosthetic osteolysis, Gluteal muscle atrophy, Iliopsoas atrophy, Muscle edema, Muscle/Tendon tear and lymphadenopathy.

Periprosthetic soft tissue was defined as abnormal fluid/ intermediate- to low-signalintensity lesion adjacent to the prosthesis on T1W and T2W images. The low signal on T2-weighted images reflects metal deposition.

Sometimes high T1 signal intensity was also seen and this may also reflects metal deposition, though haemorrhage may also present similarly. A peripheral T1 hyperintense rim may also be indicative of infection. Hence, there is always a dilemma in distinguishing juxtaarticular abscess from these reactive masses on the basis of imaging features, especially in symptomatic patients and diagnostic confidence can be achieved only by biopsy and analysis of the material.
The periprosthetic soft tissues were classified into three types based on MRI findings: Type 1 , cystic masses with wall $<3 \mathrm{~mm}$ (Fig. 1); type 2 , cystic masses with wall $>3 \mathrm{~mm}$ but less than the diameter of the cystic component (Fig.2); and type 3, predominantly solid masses (Fig. 3). ${ }^{16}$ The size of the mass and its relationship to surrounding structures was meticulously defined. Careful inspection for communication of collection with joint was done to avoid erroneously labelling a thickened noncommunicating trochanteric bursa as a "reactive mass." 17

Osteolysis was denoted as intermediatesignal-intensity marrow replacement of the hyperintense fatty marrow. The presence, location (acetabular, femur or both) as well as the size was recorded. The pathophysiology behind this periprosthetic ostelolysis was essentially the same as that described for the reactive masses.

Focal well defined area of osteolysis without surrounding edema was usually labelled as focal particle disease (Fig. 4).

Linear, long segment,fluid signal-intensity marrow replacement of $>2 \mathrm{~mm}$ width, along the bone prosthesis interface, without significant marrow edema, was suggestive of aseptic loosening of the prosthesis (Fig. 5). An irregular illdefined area of osteolysis with marrow edema was usually seen in casesof infection. Presence of enlarged lymph nodes added to the level of confidence.

The gluteus and ilio- psoas muscles were assessed for the presence of atrophy, defined as loss of volume and the presence of fatty replacement, and for the presence of muscle 


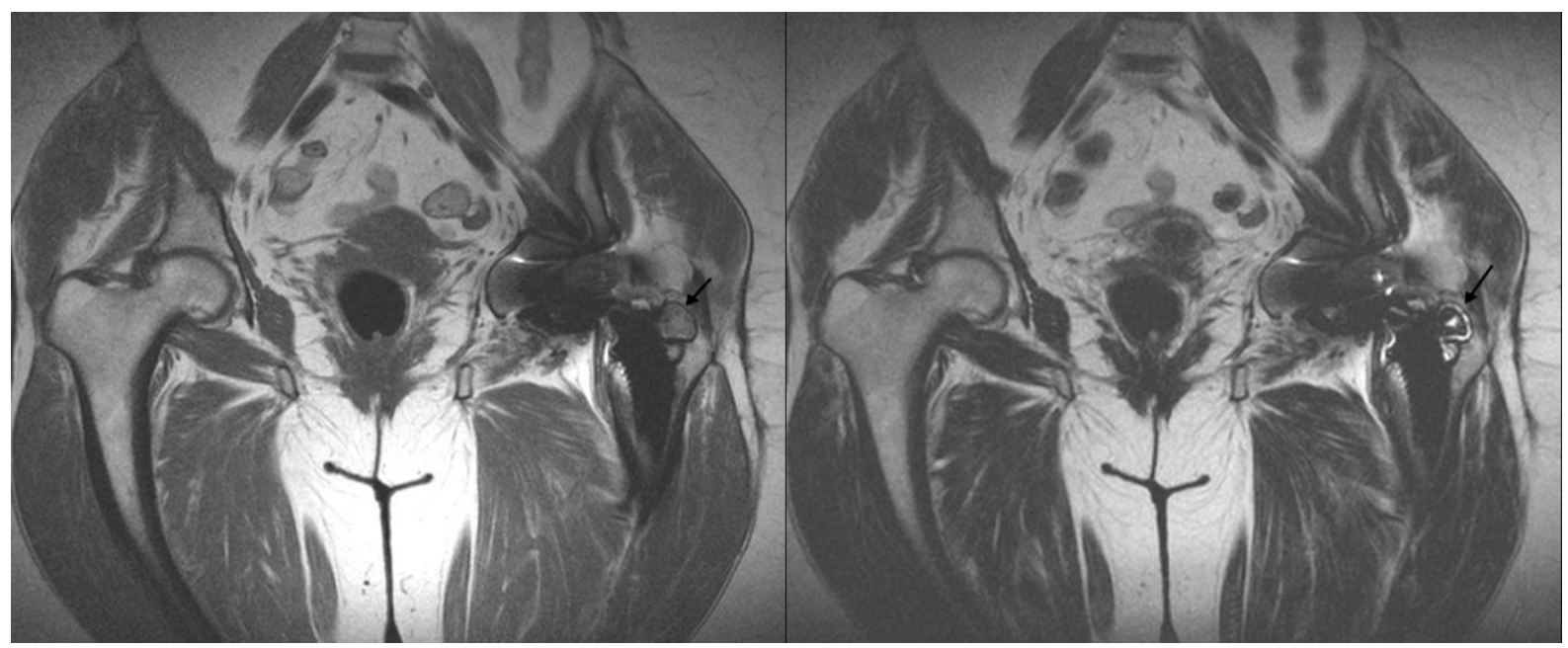

Fig. 4: Focal particle disease: Focal areas of periprostheticosteolysis in left greater trochanter.

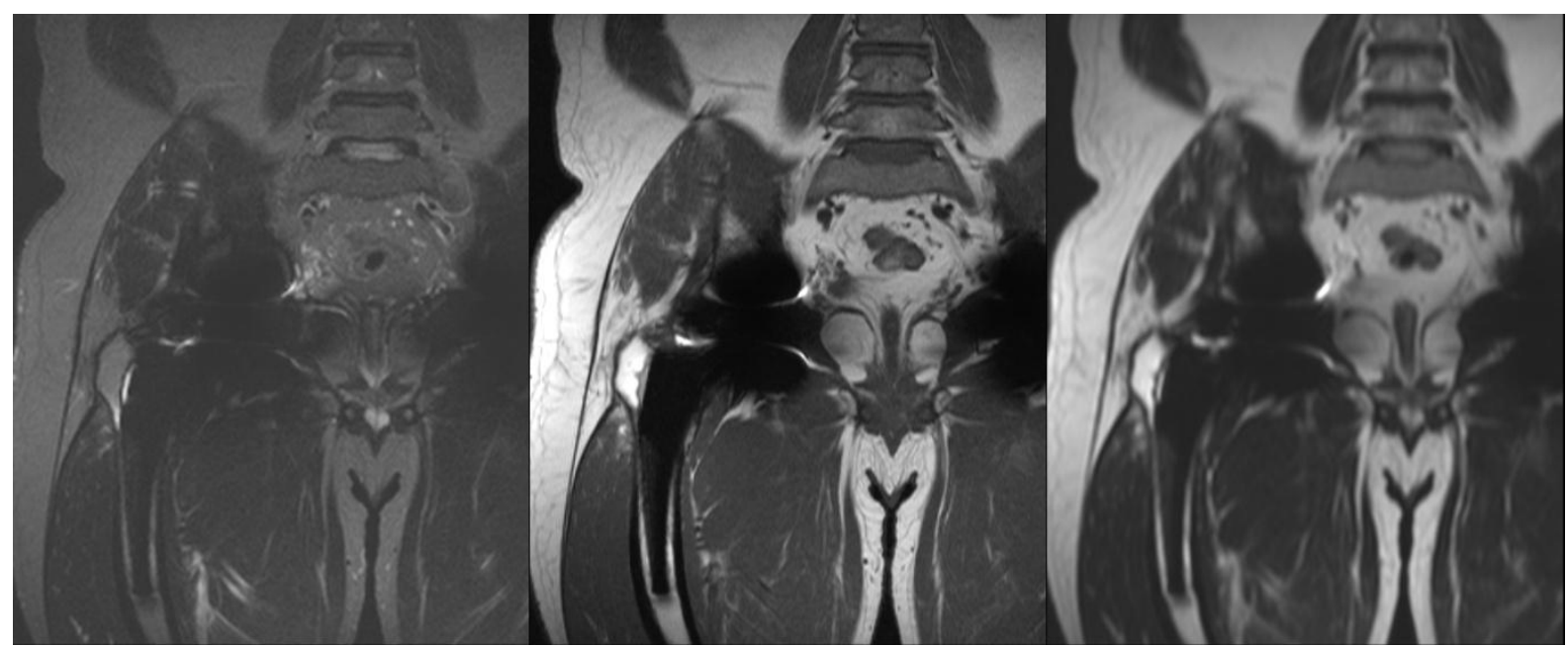

Fig. 5: Loosening: Linear fluid signal-intensity marrow replacement of $>\mathbf{2 m m}$ width, along the lateral aspect of the femoral bone prosthesis interface.

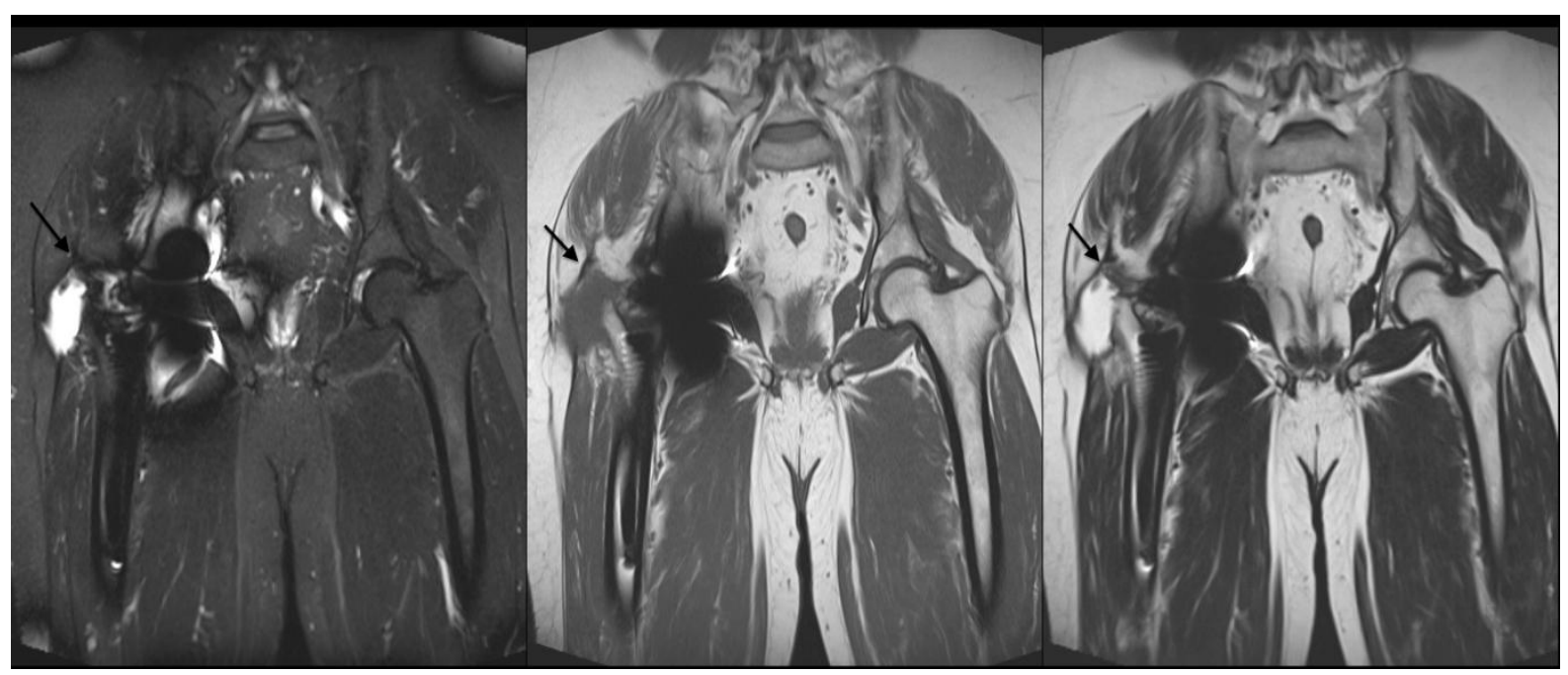

Fig. 6: Abductor tear: Tear of the tendinous insertion of the right gluteus medius. 
edema, defined as the presence of high signal intensity on inversion recovery images.

The presence or absence of a tear was graded as follows: 1, no tear; 2, partial-thickness tear; or 3, full-thickness tear (Fig. 6).

Pelvic region was assessed for enlarged lymph nodes with enlargement being defined as $>1 \mathrm{~cm}$ in short axis diameter.

\section{Results}

Diagnostic images were obtained in all patients. Of the 52 hips, 32 had total hip replacement (THR) and 20 had resurfacing. The following abnormalities were found.

Periprosthetic soft tissue mass (24), Periprosthetic osteolysis (5), Gluteal muscle atrophy - moderate to severe (15), Iliopsoas atrophy (15), Muscle edema (0), Muscle / tendon tear (1), Lymphadenopathy (5). The most commonly found abnormality was periprosthetic soft tissue in 24 hips. These are metal-induced reactive masses and are proposed to be a result of delayedhypersensitivity to the metal particles ${ }^{18} \&$ have been labeled histologically "asepticlymphocyte- dominated vascularassociated lesions" (ALVAL lesions). ${ }^{17}$

Female sex, small prosthetic cup size and poor positioning of the components are factors that possibly increase the risk of developing these reactive masses. ${ }^{19,20,21}$

These periprosthetic masses have been recognized as a major cause of revision which is required especially in patients with troublesome symptoms orlarger lesions. Correct managementof patients with smaller lesions and minorsymptoms is still uncertain because it is not known whether these lesions are likely to progress. ${ }^{17}$

Gluteal muscle atrophy - moderate to severe was appreciated in 15 hips. 15 of 52 hips had iliopsoas atrophy. 5 showed periprosthetic osteolysis of which one had features suggestive of loosening the femoral prosthesis. Lymphadenopathy was reported in 5 patients. None of the patients studied showed evidence of muscle edema.

\section{Discussion}

With the ever increasing number of MOM hip replacements world wide, the demand for prompt and early diagnosis of post arthroplasty complications has increased as well.

Radiographs and nuclear imaging, earlier the mainstays for evaluation of post arthroplasty hips, had vast limitations especially with respect to evaluation of soft tissue details which account for the symptoms in a significant number of patients. MR with its specialised metal artefact reduction sequences (MARS) is proving to be of immense help in detailed evaluation of these soft tissue complications.

Periprosthetic reactive soft tissues, the most common cause of revision is usually not detected on radiographs and picked up with relatively high sensitivity on the MR even in early stages.

MR allows early diagnosis of infection way before changes were evident on radiographs and thus allows prompt and early treatment.MRI also is more sensitive in diagnosing stress fractures. Periprosthetic osteolysis is also detected with higher 
sensitivity and specificity on MR. Differentiating between periprosthetic osteolysis and osteopenia that occurs in areas of decreased stress ("stress shielding"), is also quite straightforward with MR.

\section{Conclusion}

This study thus underlines the fact that MR on a $1.5 \mathrm{~T}$ scanner with its specialised metal artefact reduction sequences (MARS) is a viable technique to image the MoM hip and has vast potential in prompt and early diagnosis of complications in these patients.

\section{References}

1. Lee MJ, Kim S. Overcoming Artifacts from Metallic Orthopedic Implants at High-Field-Strength MR Imaging and Multidetector CT.RadioGraphics 2007;27:791-803.

2. White LM, Buckwalter KA. Technical considerations: $\mathrm{CT}$ and MR imaging in the postoperative orthopedic patient. Semin Musculoskelet Radiol 2002;6:517.

3. Guermazi A, Miaux Y, Zaim S, Peterfy CG, White D, Genant HK. Metallic artefacts in MR imaging: effects of main field orientation and strength. Clin Radiol 2003;58:322-328.

4. Mitchell DG, Cohen MS. Transverse magnetization and T2 contrast. In: Mitchell DG, Cohen MS, eds. MRI principles. 2nd ed. New York, NY: Springer-Verlag, 2000;35-47.
5. Cho ZH, Ro YM. Reduction of susceptibility artefact in gradient-echo imaging. Magn Reson Med 1992;23:193-200.

6. White LM, Kim JK, Mehta M. Complications of total hip arthroplasty: MR imaging-initial experience. Radiology 2000;215:254-262.

7. Suh JS, Jeong EK, Shin KH. Minimizing artifacts caused by metallic implants at MR imaging: experimental and clinical studies. AJR Am J Roentgenol 1998;171:1207-1213.

8. Johnston C, Kerr J, Ford S, O'Byrne J, Eustace S. MRI as a problem-solving tool in unexplained failed total hip replacement following conventional assessment. Skeletal Radiol 2007;36:955-961.

9. Eustace S, Jara H, Goldberg R. A comparison of conventional spin-echo and turbo spinecho imaging of soft tissues adjacent to orthopaedic hardware. AJR 1998;170:455-458.

10. Eustace S, Shah B, Mason M. Imaging orthopaedic hardware with an emphasis on hip prostheses. Orthop Clin North Am 1998;29:67-84

11. Potter HG, Nestor BJ, Sofka CM, Ho ST, Peters LE, Salvati EA. Magnetic resonance imaging after total hip arthroplasty: evaluation of periprosthetic soft tissue. J Bone Joint Surg Am 2004;86:1947-1954.

12. Törmänen J, Tervonen $\mathrm{O}$, Koivula A, Junila J, Suramo I. Image technique 
optimization in MR imaging of a titanium alloy joint prosthesis. J Magn Reson Imaging 1996;6:805-811.

13. Eustace S, Goldberg R, Williamson D. MR imaging of soft tissues adjacent to orthopaedic hardware: techniques to minimize susceptibility artifact. Clin Radiol 1997;52:589-594.

14. Olsen RV, Munk PL, Lee MJ. Metal artefact reduction sequence: early clinical applications. RadioGraphics 2000;20:699-712.

15. Viano AM, Gronemeyer SA, Haliloglu M, Hoffer FA. Improved MR imaging for patients with metallic implants. Magn Reson Imaging 2000;18:287-295.

16. Hauptfleisch J, Pandit H, Grammatopoulos G, Gill HS, Murray DW, Ostlere S. A MRI classification of periprosthetic soft tissue masses (pseudotumours) associated with metalon-metal resurfacing hip arthroplasty. Skeletal Radiol 2012;41:149-155.

17. Ostlere S. How to image Metal-on-Metal prostheses and their complications. AJR 2011;197:558-567.
18. Davies AP, Willert HG, Campbell PA, LearmonthID, Case CP. An unusual lymphocytic perivascular infiltration in tissues around contemporarymetal-onmetal joint replacements. J Bone JointSurg Am 2005;87:18-27.

19. Grammatopoulos G, Pandit H, Murray DW, Gill HS. Oxford Hip and Knee Group. The relationship between headneck ratio and pseudotumour formation in metal-on-metal resurfacing arthroplasty of the hip. J Bone Joint Surg Br 2010;92:1527-1534.

20. De Haan R, Campbell PA, Su EP, De Smet KA. Revision of metal-on-metal resurfacing arthroplasty of the hip: the influence of malpositioning of the components. J Bone Joint Surg $\mathrm{Br}$ 2008;90:1158-1163.

21. Glyn-Jones S, Pandit H, Kwon YM, Doll H, Gill HS, Murray DW. Risk factors for inflammatorypseudotumour formation following hip resurfacing.J Bone Joint Surg Br 2009;91:1566-1574. 